Tropical Journal of Pharmaceutical Research March 2016; 15 (3): 453-459

ISSN: $1596-5996$ (print); 1596-9827 (electronic)

(C) Pharmacotherapy Group, Faculty of Pharmacy, University of Benin, Benin City, 300001 Nigeria.

All rights reserved.

Available online at http://www.tjpr.org

Original Research Article

http://dx.doi.org/10.4314/tjpr.v15i3.4

\title{
Ixeris dentata (Thunb) Nakai Ethylacetate Extract Attenuates Sterol Regulatory Element-Binding Proteins-1c via AMP-Activated Protein Kinase Activation
}

\author{
Jong-Bo Kim ${ }^{1}$ and Hyun Kang ${ }^{2 *}$ \\ ${ }^{1}$ Department of Biotechnology, Research Institute (RIBHS) and College of Biomedical and Health Science, Konkuk University, \\ Chungju, Chungbuk, 380-701, ${ }^{2}$ Department of Medical Laboratory Science, College of Health Science, Dankook University, \\ Cheonan-si, Chungnam, 330-714, Republic of Korea
}

*For correspondence: Email: hkang@dankook.ac.kr; Tel: 82-41-550-1452; Fax: 82-41-559-7934

Received: 6 November 2015

Revised accepted: 13 February 2016

\begin{abstract}
Purpose: To investigate the molecular mechanisms underlying the role of Ixeris dentataa extract (IDE) in the prevention of high glucose-induced lipid accumulation in human HepG-2 hepatocytes.

Methods: IDE extract was prepared by maceration in ethyl acetate. Its fractionation was carried out by column chromatography. HepG-2 cells were pretreated with various concentration of IDE $(0,10,20,40$ and $80 \mu \mathrm{g} / \mathrm{mL}$ ) and then treated with serum-free medium with normal glucose $(5 \mathrm{mM})$ for $1 \mathrm{~h}$, followed by exposure to high glucose (30 $\mathrm{mM}$ D-glucose) for $24 \mathrm{~h}$. Cell viability and cytotoxicity parameters were measured using lactate dehydrogenase (LDH) and MTT assay while triglyceride and total cholesterol levels were evaluated using respective enzymatic reagent kits. Protein expressional levels were analyzed by Western blotting.

Results: IDE did not influence the cell viability (up to $200 \mu \mathrm{g} / \mathrm{mL}$ ) and did not show any signs of cytotoxicity (up to $80 \mu \mathrm{g} / \mathrm{m} / \mathrm{L}$. IDE significantly attenuated lipid accumulation in human HepG2 hepatocytes when exposed to high glucose (30 mM D-glucose) in a dose-dependent manner $(p<0.05$, 0.01 and 0.001 at 20, 40 and $80 \mu \mathrm{g} / \mathrm{mL}$ concentrations, respectively). Nile red staining showed that 10 , 20,40 and $80 \mu \mathrm{g} / \mathrm{mL}$ concentrations of IDE reduced lipid accumulation by 23.4, $34.8(p<0.05), 46.5$ ( $p$ $<0.01)$ and $53.2 \%(p<0.001)$, respectively. The increased levels of triglycerides and total cholesterol were also attenuated by IDE $(p<0.001$ at $80 \mu \mathrm{g} / \mathrm{mL})$. Further, IDE attenuated the expression of fatty acid synthase and sterol regulatory element-binding protein-1. Adenosine monophosphate-activated protein kinase was also activated by IDE treatment when exposed to high glucose level (30 mM Dglucose) in human HepG2 hepatocytes.

Conclusion: The findings indicate that IDE exerts hypolipidemic effect by inhibiting lipid biosynthesis, mediated via AMPK signaling. This probably explains the extract's beneficial effect in various inflammation disorders.
\end{abstract}

Keywords: Ixeris dentata extract, Lipogenesis, AMP-activated protein kinase, Sterol regulatory element-binding protein 1-1, Anti-obesity, HepG-2 cell

Tropical Journal of Pharmaceutical Research is indexed by Science Citation Index (SciSearch), Scopus, International Pharmaceutical Abstract, Chemical Abstracts, Embase, Index Copernicus, EBSCO, African Index Medicus, JournalSeek, Journal Citation Reports/Science Edition, Directory of Open Access Journals (DOAJ), African Journal Online, Bioline International, Open-J-Gate and Pharmacy Abstracts

\section{INTRODUCTION}

Ixeris dentata (ID) is a well-known Korean traditional herb and has been commonly used in various diseases including respiratory and cardiovascular systems in Korea [1]. Since early times, both shoots and roots of ID have been used as plant-based foods such as kimchi and salad in Korea, and ID juice has been used to treat adult diseases [2]. More recently, secondary 
metabolites of ID have been known to possess anticancer, antistress, antiallergic, and antioxidant properties. Thus, ID is getting great interest as one of health functional medicinal plants [3]. However, the effect of an ethylacetate extract of ID and its molecular mechanism on lipid metabolism has not been elucidated.

Abnormal lipid and lipoprotein metabolism are known to result in dyslipidemia such as elevation of plasma cholesterol and/or TGs and decreased levels of high-density lipoprotein (HDL). Fatty liver disease is related to fat accumulation, high triacylglycerol, low high-density lipoprotein cholesterol (HDL-C), high low-density lipoprotein cholesterol (LDL-C), and insulin resistance [4].

A number of proteins associated with dyslipidemia have been reported to play important roles in lipogenesis, including fatty acid synthase (FAS), sterol regulatory element binding proteins (SREBPs) [2]. FAS are a multiple enzyme complex that catalyzes the synthesis of palmitate from acetyl-C glucose and malonyl-C glucose. Expression of FAS is regulated by multiple transcription factors. SREBPs, a transcription factor family, regulate the expression of enzymes for the synthesis of fatty acid, cholesterol, triacylglycerol, and phospholipids, suggesting that the protein family plays a central role in energy homeostasis via modulation of glycolysis, lipogenesis and adipogenesis.

Adenosine monophosphate-activated protein kinase (AMPK) is a serine threonine kinase comprising a heterotrimeric complex and regarded as an energy sensor in most tissues. AMPK regulates cellular lipid metabolism by increasing fatty acid oxidation and constitutive lipoprotein exocytosis [5]. In the liver, activated AMPK phosphorylates and inactivates the ratelimiting enzymes of lipogenesis such as ACC, suggesting that AMPK/ACC signaling plays a critical role in hepatic lipid homeostasis [6].

In previous study, ID contained approximately 4 $\mathrm{mg} / \mathrm{g}$ of caffeic acid (3,4-dihydroxy cinamic acid) [2]. Approximately 20 types of sesquiterpene and other compounds have been isolated from ID. It has been reported that its main compounds are luteolin, luteolin 7-O-glucuronide, caffeic acid, chlorogenic acid, and guaiane sesquiterpene lactones [7,8]. Among these main compounds, inhibitory effects of luteolin, chlorogenic acid, and tectroside on allergic inflammation had already been reported. The major active components of ID are phenolic constituents $[2,3]$.
This study aimed to examine whether ID extract (IDE) could suppress the hepatic lipid induced by high glucose and regulate lipid metabolism.

\section{EXPERIMENTAL}

\section{Plant material}

The whole plant of $I$. dentata were collected on May 2014 at the herbarium were obtained from the local market, Seoul, South Korea. The fruits were authenticated by Professor Eun-Young Choi, a taxonomist, Konkuk University, Korea, and a voucher specimen (IDKU2014 was kept in the university herbarium for future reference. All reagents used in this study were of the highest commercial grade available.

\section{Preparation of the ID extract}

We employed a previous preparation method for this extract [9]. Briefly, $500 \mathrm{~g}$ of the fruit were ground in a mixer and defatted three times with three volumes of $80 \%$ ethanol. The residue was extracted with absolute ethanol (EtOH) at 1:10 ratio $(\mathrm{w} / \mathrm{v})$ for $2 \mathrm{~h}$ on a heated mantle at $70-80$ ${ }^{\circ} \mathrm{C}$. The supernatant was filtered and concentrated in a rotatory evaporator at $50{ }^{\circ} \mathrm{C}$. The ethanol extract obtained $(180 \mathrm{~g})$ was resuspended in water:EtOH $(9: 1, \quad \mathrm{v} / \mathrm{v})$ and partitioned successively with $n$-hexane, ethyl acetate (EA) and n-butanol to obtain final yields of $19.4,52$ and $27.27 \%$, respectively. EA fraction of olive pulp fruit extract was dissolved in sterile distilled water and filtered on $0.22 \mu \mathrm{m}$ filters before use.

\section{Cell culture and treatment}

Human HepG-2 hepatocytes obtained from the American Type Culture Collection (Rockville, MD) were cultured in Dulbecco's modified Eagle's medium (DMEM) containing normal glucose (5.5 mM d-glucose), supplemented with $10 \%$ heat-inactivated foetal bovine serum (FBS; Invitrogen). Cells were cultured in a humidified $5 \% \mathrm{CO} 2$ incubator at $37^{\circ} \mathrm{C}$ in complete medium supplemented with $10 \%$ FBS to $80 \%$ confluence for use in the assays.

IDE was dissolved in dimethyl sulfoxide (DMSO) and added directly to serum-free culture medium. The final concentration of DMSO did not exceed $0.1 \%(\mathrm{v} / \mathrm{v})$ and did not affect cell viability nor produced cytotoxicity. A cell model of the high glucose induced accumulation of hepatic lipids was prepared by exposing HepG-2 cells to 30 $\mathrm{mM}$ of glucose for $24 \mathrm{~h}$. 


\section{Measurement of cell viability and cytotoxicity}

Cells were cultured at $37{ }^{\circ} \mathrm{C}$ in medium containing $10 \%$ FBS at a density of $4 \times 10^{4}$ cells $/ 500 \mu \mathrm{L}$ in 48-well plates. After $24 \mathrm{~h}$, the growth medium was replaced with serum-free medium and the cells were treated with different concentrations of IDE $(10-100 \mu \mathrm{g} / \mathrm{mL})$ or an equal volume of DMSO for $24 \mathrm{~h}$ at $37^{\circ} \mathrm{C}$. The culture medium was used in a lactate dehydrogenase (LDH) assay, with the absorbance at $490 \mathrm{~nm}$ measured using a microplate reader (Varioskan; Thermo Electron, Waltham, MA). Cytotoxicity was calculated based on the absorbance relative to that of the control. Cells were treated with MTT solution (final concentration, $0.5 \mathrm{mg} / \mathrm{mL}$ ) for $1 \mathrm{~h}$. The dark blue formazan crystals that formed in intact cells were solubilized with DMSO, and the absorbance at $570 \mathrm{~nm}$ was measured with a microplate reader. Cell viability was calculated based on the absorbance of the IDE-treated cells relative to that of control vehicle-treated cells.

\section{Nile red stain}

Human HepG2 hepatocytes were seeded in a 6well plate $\left(3 \times 10^{6}\right.$ cells/well $)$ and treated with glucose $(30 \mathrm{mM})$ with indicated concentrations of IDE for $24 \mathrm{~h}$. The cells were washed twice with PBS and fixed with $4 \%$ formaldehyde in PBS for $1 \mathrm{~h}$ and then stained with $1 \mu \mathrm{g} / \mathrm{mL}$ Nile red for 30 min at room temperature. After staining, the distribution of lipid in cells was immediately analyzed by a FACScan flow cytometer (Becton Dickinson, Mountain View, CA, USA). Lipidbound Nile red fluorescence was detected using inverted fluorescence microscopy.

\section{Determination of triglyceride and total cholesterol levels}

The intracellular triglyceride and total cholesterol contents were measured in cell lysates and expressed as micrograms of lipid per milligram of cellular protein. HEPG-2 cell were seeded in 100-mm culture dishes. Twenty-four hours after treatment, the cells were homogenized in $1 \mathrm{~mL}$ of PBS. The homogenates were extracted with $3 \mathrm{ml}$ of chloroform and methanol $(2: 1, v / v)$. The mixture was vortexed vigorously, allowed to separate into two phases, and centrifuged (3000 rpm, $10 \mathrm{~min}, 4^{\circ} \mathrm{C}$ ). An aliquot of the organic phase was evaporated under nitrogen gas until dry. The resulting pellet was dissolved in PBS containing $1 \%$ Triton $X-100$, and the protein concentration was measured. The intracellular triglyceride and total cholesterol contents were determined using an enzymatic reagent kit (Asan Pharm. Co., Seoul, Korea) at 550 and 500 nm, respectively, and normalized to the protein concentration.

\section{Western blot analysis}

Human HepG-2 were seeded in a $10 \mathrm{~cm}$ dish (1 $\times 10^{6}$ cells/well) and treated with glucose (30 $\mathrm{mM}$ ) at indicated concentrations of IDE for $24 \mathrm{~h}$. The proteins of the cells were harvested in a cold radio immunoprecipitation assay (RIPA) buffer (1 $\%$ NP-40, 50 mM Tris-base, $0.1 \%$ SDS, $0.5 \%$ deoxycholic acid, $150 \mathrm{mM} \mathrm{NaCl}, \mathrm{pH}$ 7.5). Equal amounts of protein samples were subjected to SDS-polyacrylamide gel electrophoresis and electro-transferred to nitrocellulose membranes (Millipore, Bedford, MA, USA). The membranes were blocked with $5 \%$ nonfat milk powder with $0.1 \%$ Tween-20 in TBS and then incubated with the first antibody at $4{ }^{\circ} \mathrm{C}$ overnight. Thereafter, membranes were washed three times with $0.1 \%$ Tween-20 in PBS and incubated with the secondary antibody to anti-mouse horseradish peroxidase (GE Healthcare, Little Chalfont and Buckinghamshire, UK). Antibodies against AMPK and phospho-AMPK were purchased from Cell Signaling Technology (Beverly, MA, USA). FAS and SREBP-1c antibodies were obtained from Santa Cruz Biotechnology (Santa Cruz, CA, USA). Band detection was revealed by enhanced chemiluminescence using ECL Western blotting detection reagents and exposed ECL hyper film in FUJIFILM LAS-3000 (Tokyo, Japan).

\section{Statistical analysis}

Statistical analysis was performed using SAS program (SAS 9.2, SAS Institute Inc., USA). All measurements were carried out in quintuple Analysis of variance (ANOVA) was used to analyze the data followed by Duncan's multiple range test and t-test. $P<0.05$ was considered statically significant.

\section{RESULTS}

\section{Effect of IDE on HepG-2 cell viability}

We first determined the concentration dependence of the cytotoxic effects of IDE by directly adding IDE to the culture medium at various concentrations $(10 \sim 100 \mu \mathrm{g} / \mathrm{ml})$ for $24 \mathrm{~h}$, and the cell viability was measured using MTT assays (Fig 1).

IDE also showed no signs of cytotoxicity at indicated concentrations $(10 \sim 100 \mu \mathrm{g} / \mathrm{ml})$ under normal glucose and high glucose conditions in HepG-2 cell (Fig 2). Considering the results we 


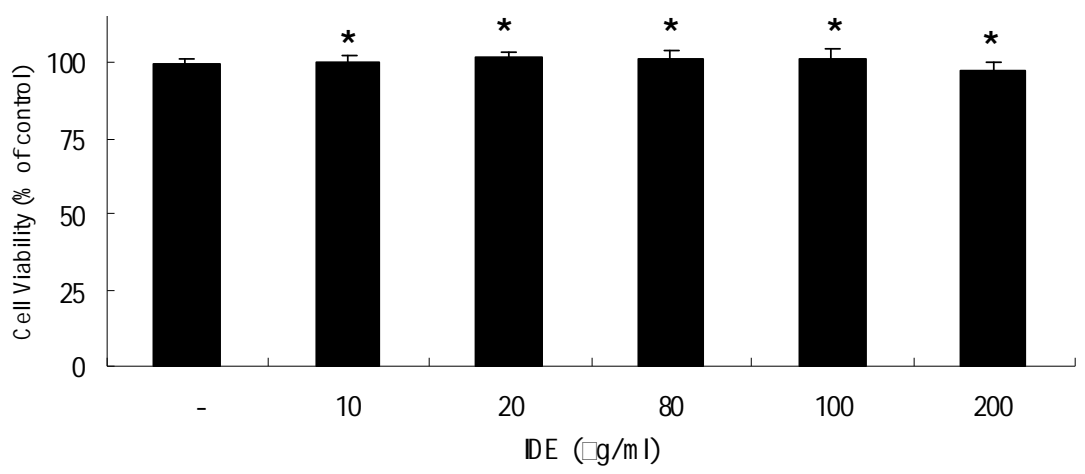

Figure 1: Effect of IDE on overall cell viability in human HepG-2 hepatocytes. Cell viability was assessed by MTT assay and expressed as percentage of the vehicle controls. Data expressed are means \pm SD of three independent experiments conducted in triplicate. " No significant difference exists when compared to control group. IDE: ID extract

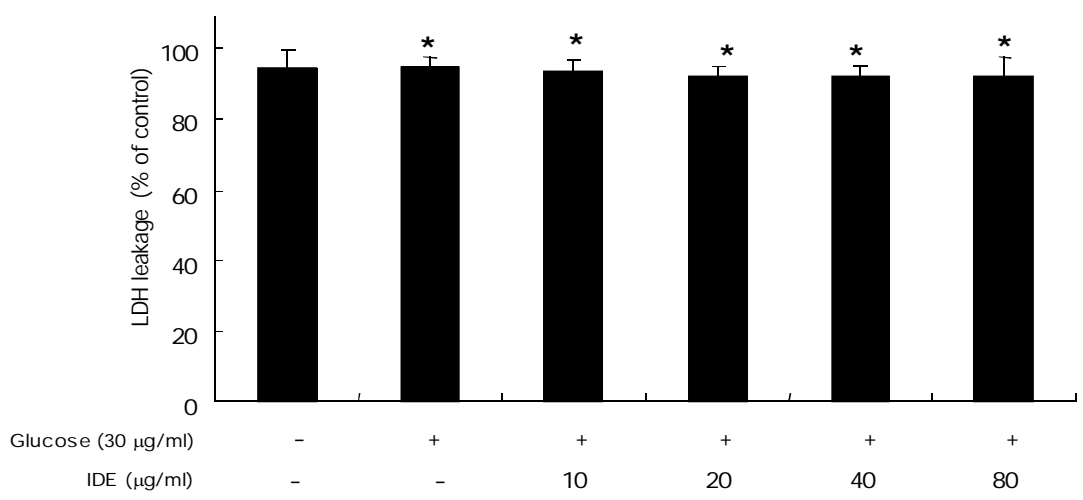

Figure 2: Effect of IDE on HepG2 cell cytotoxicity. Cytotoxic effects of IDE on HepG-2 cell under high glucose conditions, respectively. Cell cytotoxicity were assessed by LDH assay and expressed as percentages of the vehicle controls. Data expressed are means $\pm \mathrm{SD}$ of three independent experiments conducted in triplicate. ${ }^{*}$ No significant difference exists when compared to control groups. IDE: ID extract

used IDE in the range of $10 \sim 80 \mu \mathrm{g} / \mathrm{ml}$, respectively for further experiments.

\section{IDE attenuates lipid accumulation in human HepG-2 hepatocytes}

To assess the inflammatory responses of IDE in vitro, HepG-2 cell were pretreated with various concentration of IDE $(10,20,40$ and $80 \mu \mathrm{g} / \mathrm{mL})$ and treated with serum-free medium with normal glucose $(5 \mathrm{mM})$ for $1 \mathrm{~h}$, followed by exposure to high glucose (30 mM D-glucose) for $24 \mathrm{~h}$. Nile red staining showed that $10,20,40$, and 80 $\mu \mathrm{g} / \mathrm{mL}$ concentrations of IDE reduced lipid accumulation by $23.4,34.8,46.5$ and $53.2 \%$, respectively (Fig. 3A).

The effects of IDE on the triglyceride and total cholesterol levels showed that 20,40 , and 80 $\mu \mathrm{g} / \mathrm{mL}$ of IDE reduced the triglyceride levels significantly, in the high glucose-treated cells by $38.9 \%, 55.6 \%$, and $63.4 \%$, respectively (Fig. 3B). IDE at indicated concentrations also decreased the cholesterol content of HepG-2 cell incubated in high glucose for $24 \mathrm{~h}$ by $40.1,52.1$, and $61.2 \%$, at 20, 40, and $80 \mu \mathrm{g} / \mathrm{mL}$ concentrations, respectively (Fig. 3C). These results indicate that IDE can attenuate high glucose-mediated lipid accumulation in human HepG-2 hepatocytes.

\section{IDE inhibits lipid accumulation via SREBP-1c inactivation in human HepG-2 hepatocytes}

We examined the status of SREBP-1c in high glucose-induced HepG-2 cell in the presence or absence of IDE at indicated concentrations. SREBP-1 protein levels were increased markedly in cells treated with high glucose $(30 \mathrm{mM})$ for 12 h (Fig 4, upper panel).

\section{IDE promotes AMPK phosphorylation in human HepG-2 hepatocytes}

The phosphorylation of AMPK was determined in HepG-2 cell under high glucose conditions by Western blotting. HepG-2 cell cultured in serumfree medium with normal glucose $(5 \mathrm{mM})$ were 

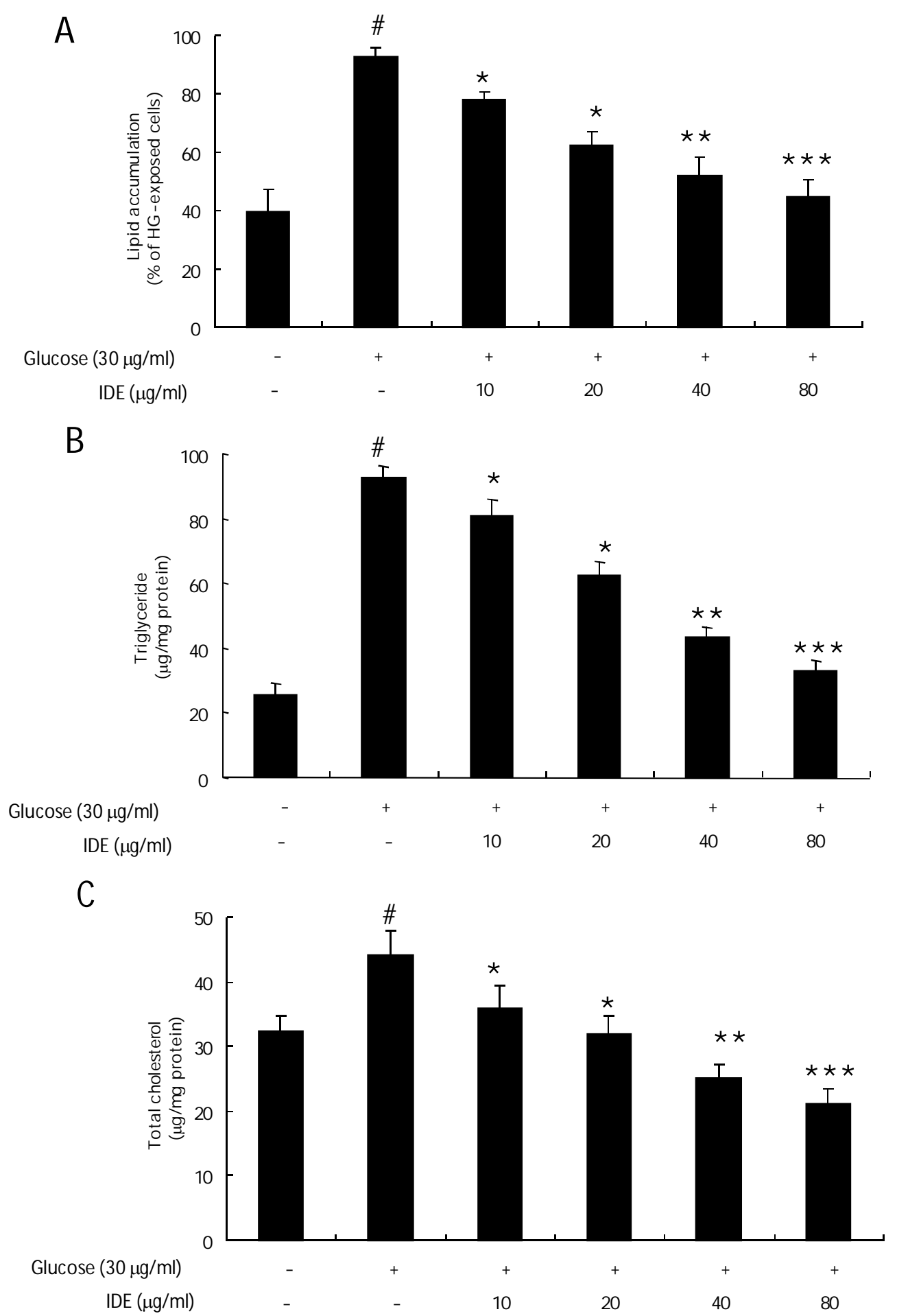

Figure 3: IDE attenuates high glucose-induced lipid accumulation in human HepG-2 hepatocytes. A: Effect of IDE on high glucose-induced lipid accumulation in human HepG-2 hepatocytes. B: Intracellular triglyceride and C: total cholesterol contents in cell lysates. Data shown are the means \pm SD of three independent experiments performed in triplicate; ${ }^{\#} p<0.001$ vs. control; ${ }^{*} p<0.05$, ${ }^{* *} p<0.01$ and ${ }^{* * *} p<0.001$ vs. high glucose-exposed cells, respectively; IDE = ID extract

pretreated with IDE (20, 40 and $80 \mu \mathrm{g} / \mathrm{mL})$ for 1 $\mathrm{h}$, followed by exposure to high glucose $(30 \mathrm{mM})$ for an additional 2 h. Although the phosphorylation of AMPK was significantly suppressed in cells treated with high glucose (30 $\mathrm{mM})$, IDE dose-dependently restored the phosphorylation of AMPK in IDE-pretreated cells (Fig 5). 


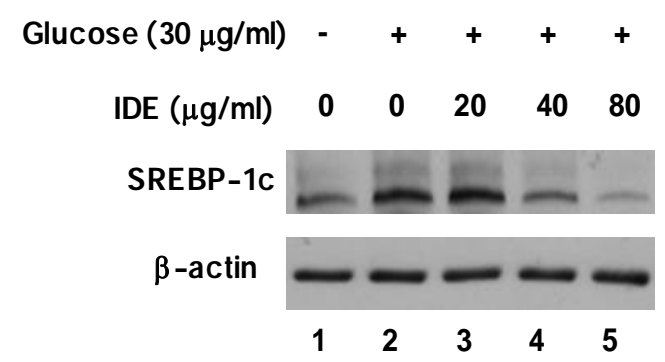

Figure 4: Effect of IDE treatment on SREBP-1C protein expression in human HepG-2 hepatocytes. SREBP-1c protein levels in the cell lysates were detected by Western blot analysis. All experiments were performed in triplicate. IDE: ID extract

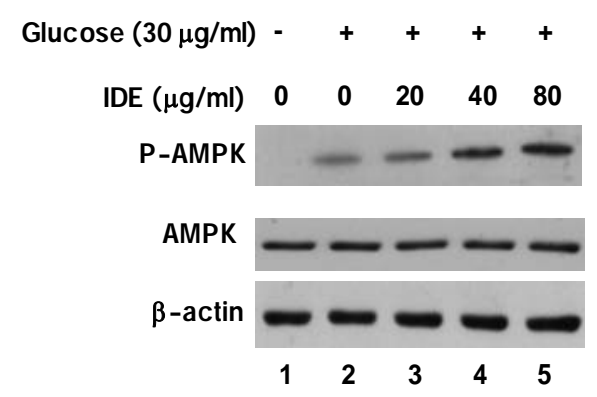

Figure 5: Effect of IDE treatment on pAMPK/AMPK protein expression in human HepG2 hepatocytes. The protein levels of $\mathrm{PAMPK} / \mathrm{AMPK}$ in the cell lysates were detected by Western blot analysis. $\beta$-actin was used as an internal control. All experiments were performed in triplicate. IDE: ID extract

\section{DISCUSSION}

The species, Ixeris dentata (ID) is a perennial herb of Asteraceae which was widely distributed and cultivated in Northeastern Asia [1]. The flowering time is from May to July and the flowers are ligulate and yellow [1]. This plant grows to height of about $25-50 \mathrm{~cm}$ [2]. The young sprouts of this species had been used as a famous bitter appetizing vegetable in Korea.

The plant has been used for the treatment of indigestion, pneumonia, hepatitis, contusion and tumor and various gastroenteric troubles in Korea [3]. According to recent research, ID has been proved to have a hypocholesterolaemic effect and an antioxidant activity, as well as ID is known to have aliphatics, triterpenoids and sesquiterpene glycosides in its composition $[7,8]$. Studies on the roots revealed the effects of an antioxidant, anti-mutagenicity and an anticancer. The methanol extract was most effective in the anticancer activity, inhibiting the growth of MG-63 cells by more than ninety percent [8]. Also, the effect of ID on the cardiovascular system in hyperlipidemic rats was examined [8] and also caused prevention of neurodegenerative diseases [9,10]. It has also been used for the treatment of allergic diseases as a folk therapy in Korea. The hepatic hypolipidemic mechanism of Ixeris dentata extract (IDE) showed high relevant expression of lipogenic enzyme, cholesterol biosynthesis and TG biosynthesis in HepG-2 cell. Taken together, these observations suggest that the neuro-inflammatory responses of IDE may require the AMPK signaling pathway. Activation of AMPK leads to regulation of a number of downstream targets involved in lipid metabolism [5]. The regulation of AMPK activation and the most effective constituent in IDE need to be elaborated.

Several natural products from plant origin have been suggested to be capable of preventing obesity via regulating hyperlipidemia and reducing adipose tissue mass, thus suppressing the occurrence of the metabolic, hepatic, and cardiovascular alterations associated with obesity $[10,11]$. Many studies have indicated that antioxidant phenolic compounds have been used in cellular and animal models for the treatment of obesity and neuro-inflammatory responses $[13,14]$. Polyphenols from blueberries and/or strawberries reduced the body weight and body fat of rats fed a high-fat diet [15]. Polyphenol rich antioxidants reduced hyperglycemia efficiently in type 2 diabetic mice via inducing AMPK expression in the liver and then resulted in significantly decreased liver and serum lipid contents [16].

The recent report showed that IDE possessed antioxidant properties [9, 10]. ID has been used for the treatment of mithridatism, calculous, indigestion, pneumonia, hepatitis, and tumors in Korea, China, and Japan [1]. It has been reported that ID has neuro-protective effects [10], anti-diabetic effects [11], protection effects against colitis and skin inflammation [2,3], and anti-allergic effects $[10,11]$. In this study, the effect of the IDE etylacetate extract on lipid metabolism via AMPK signaling was investigated in human HepG-2 hepatocytes. Earlier studies revealed that ID phenols possessed strong antioxidant compounds [18]. The ethyl acetate fraction present in ID might be responsible in exhibiting such potent antioxidant and antiinflammatory effects.

\section{CONCLUSION}

The present study provides evidence that IDE plays a significant role in reducing HepG2 cellular lipid accumulation by mediating AMPK signaling, thereby inhibiting expression of SREBP-1c and further suppressing TG and 
cholesterol synthesis. The antioxidant potential of IDE might partly be responsible for this, suggesting an active role for IDE in preventing non-alcoholic fatty liver disease. Thus, the plant material has the potential to be developed to yield an anti-inflammatory agent.

\section{ACKNOWLEDGEMENT}

This study was supported by Research Fund of Dankook University in 2014.

\section{REFERENCES}

1. Ahn EM, Bang MH, Song MC, Park MH, Kim HY, Kwon BM. Cytotoxic and ACAT-inhibitory sesquiterpene lactones from the root of Ixeris dentata forma albiflora. Arch Pharm Res 2006; 29: 937-941.

2. Kim DS, Ko JH, Jeon YD, Han YH, Kim HJ, Poudel A. Ixeris dentata NAKAI Reduces Clinical Score and HIF-1 Expression in Experimental Colitis in Mice. Evid Based Complement Alternat Med 2013; 67: 1281-1289.

3. Kim SB, Kang OH, Joung DK, Mun SH, Seo YS, Cha MR. Anti-inflammatory effects of tectroside on UVBinduced HaCaT cells. Int $\mathrm{J} \mathrm{Mol} \mathrm{Med} \mathrm{2013;} \mathrm{31:} 1471$ 1476.

4. Moller D, Kaufman KD. Metabolic syndrome: a clinical and molecular perspective. Annu Rev Med 2005; 56: 4562.

5. Puljak LV, Parameswara S, Dolovcak, SL, Waldrop D, Emmett V, Esser JG, Kilic G. Evidence for AMPKdependent regulation of exocytosis of lipoproteins in a model liver cell line Exp. Cell Res 2008; 314: 2100 2109.

6. Lagouge M, Argmann C, Gerhart-Hines Z. Resveratrol improves mitochondrial function and protects against metabolic disease by activating SIRT1 and PGC-1alpha. Cell 2006; 127: 1109-1122.

7. Cha MR, Choi YH, Choi CW, Yoo DS, Kim YS, Choi SU. New guaiane sesquiterpene lactones from Ixeris dentata. Planta Med 2011; 77: 380-382.
8. Karki S, Park HJ, Nugroho A, Kim EJ, Jung HA, Choi JS. Quantification of major compounds from Ixeris dentata, Ixeris dentata Var. albiflora, and Ixeris sonchifolia and their comparative anti-inflammatory activity in lipopolysaccharide-stimulated RAW 264.7 cells. J Med Food 2015; 18: 83-94.

9. Kim MS, Koppula S, Jung SH, Kim JY, Lee HR, Lee SR, Park YD, Lee KA, Park TK, Kang $H$. Ixeris dentata Extract Exhibits Potent Antioxidant Activity and Attenuates Neuro-inflammatory Responses in Lipopolysaccharide- Stimulated Microglial Cells. Trop J Pharm Res 2013; 12: 357-362.

10. Oh SH, Sung TH, Kim MR. Ixeris dentata extract maintains glutathione concentrations in mouse brain tissue under oxidative stress induced by kainic acid. $J$ Med Food 2003; 6: 353-358.

11. Lee HY, Lee GH, Kim HK, Kim SH, Kp P, Chae HJ. Ixeris dentata-induced regulation of amylase synthesis and secretion in glucose-treated human salivary gland cells. Food Chem Toxicol 2013; 62: 739-749.

12. Yi JM, Hong SH, Lee HJ, Won JH, Kim JM, Jeong DM. Ixeris dentata green sap inhibits both compound 48/80induced anaphylaxis-like response and $\mathrm{IgE}$ mediated anaphylactic response in murine model. Biol Pharm Bull 2002; 25: 5-9.

13. Hsu CL, Yen GC. Phenolic compounds: Evidence for inhibitory effects against obesity and their underlying molecular signaling mechanisms. Mol Nutr Food Res 2008; 52: 53-61.

14. González R, Ballester I, López-Posadas R, Suárez MD, Zarzuelo A, Martínez-Augustin O, Sánchez de Medina F. Effects of flavonoids and other polyphenols on inflammation. Crit Rev Food Sci Nutr 2011; 51: 331-362.

15. Prior RL, Wu X, Gu L, Hager T, Hager A, Wilkes $S$, Howard L. Purified berry anthocyanins but not whole berries normalize lipid parameters in mice fed an obesogenic high fat diet Mol. Nutr Food Res 2009; 53 : 1406-1418.

16. Takikawa M, Inoue S, Horio F, Tsuda, T. Dietary anthocyanin-rich bilberry extract ameliorates hyperglycemia and insulin sensitivity via activation of AMP-activated protein kinase in diabetic mice. J Nutr 2010; 140: 527-533. 